\title{
RPBP: Rapid-Prototyped Remote-Brain BiPed with 3D Perception
}

\author{
Marsette Vona* and Dimitrios Kanoulas*
}

\begin{abstract}
This paper provides the design of a novel open-hardware mini-bipedal robot, named Rapid-Prototyped Remote-Brain BiPed (RPBP), that is developed to provide a low-cost and reliable platform for locomotion and perception research. The robot is made of customized 3D-printed material (ABS plastic) and electronics, and commercial Robotics Dynamixel MX-28 actuators, as well as visual RGB-D and IMU sensing systems. We show that the robot is able to perform some locomotion/visual-odometry tasks and it is easy to switch between different feet designs, providing also a novel Center-ofPressure (CoP) sensing system, so that it can deal with various types of terrain. Moreover, we provide a description of its control and perception system architecture, as well as our opensource software packages that provide sensing and navigation tools for locomotion and visual odometry on the robot. Finally, we briefly discuss the transferability of some prototype research that has been done on the developed mini-biped, to half or fullsize humanoid robots, such as COMAN or WALK-MAN.
\end{abstract}

\section{INTRODUCTION}

Humanoid robots have started playing a significant role in human's life, and their usability is emerging both for indoors and outdoor tasks. Replacing humans in tasks such as housekeeping or even more importantly, hazardous ones after a natural/human-made disaster, makes humanoid systems very valuable. Bipedal locomotion is a very important, but challenging, aspect of their usability, when navigating in realworld environments. For this reason, a lot of ongoing research is focused on the particular topic and the development of such platforms is crucial.

Since the 1970s, the design of legged bipedal humanoid robots is emerging in robotics. Since then, a variety of humanoid robots have been used, with impressive capabilities both indoors and outdoors. For instance, some of the most famous full and half-size humanoids that were developed are the following. The WABOT humanoid [1], from Waseda University in Japan, was one of the first humanoid robots that provided locomotion/manipulation control, as well as a vision/audio system. Its design played an important role and worked as a template for the following humanoid designs. Since then, more impressive powered robots have been developed with high efficiency performance, such as ASIMO [2] from Honda in Japan, and the HRP series (2L-4C) [3] from AIST and Kawada Industries Inc. in Japan. More recently, a series of Hubo humanoids, named KHR-1/2/3 [4], were developed from KAIST, winning also recently the DARPA

Marsette Vona was with the CCIS, Northeastern University, Boston, Massachusetts. vona@ jpl.nasa.gov.

Dimitrios Kanoulas is with the Humanoid and Human-Centered Mechatronics Department, Istituto Italiano di Tecnologia (IIT), Via Morego 30, 16163, Genova, Italy. Dimitrios. Kanoulaseit.it.

*Both authors contributed equally to this work

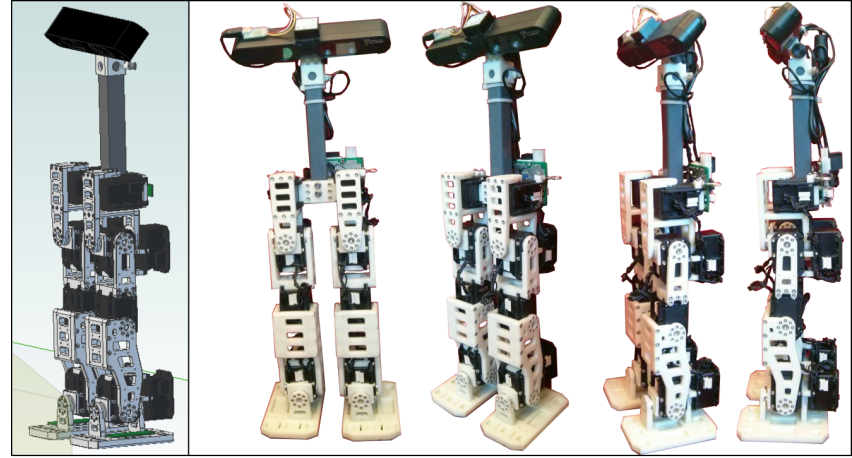

Fig. 1. RPBP: the Rapid-Prototyped BiPedal robot in simulation (left) and the physical in different angles.

Robotic Challenge (DRC) 2015, while Waseda University designed Wabian-2R with improved energy efficiency [5]. Other important electric or hydraulic full and half-size humanoids, include the LOLA/Johnnie robot [6], the iCub [7] and cCub/COMAN [8] half-size humanoids, the full-size Valkyrie humanoid [9], TORO [10] and M2V2 biped [11], JAXON [12], Hydra [13], Escher [14], TALOS [15], and WALK-MAN [16] humanoids. Several other companies have also presented very impressive humanoid or bipedal systems, such as SHAFT, Robotis THORMANG, Agility Robotics Cassie biped, and Boston Dynamics PETMAN/ATLAS.

All these full and half-size robots are impressive, but complex mechanical systems, that are designed for real scenarios. Nevertheless, as prototypes, their fragility and the sensibility in breaking (e.g., actuators, sensors, electronics) is big, with a large financial and temporal cost. Moreover, there is some research, e.g. on humanoid falling [17], or locomotion on rough terrain [18], that may cause big damages to the robot and in the same time be also dangerous for humans. Usually, these robots need more than one person to operate, for instance, holding emergency buttons, the crane, controlling the robot, etc. From our experience with the WALK-MAN humanoid during the DRC 2015, most of the research and development took place in simulation (where the transferability of the developed methods to the real robot is not always straightforward) or in smaller simpler robots, before moving to the full-size system. For this reasons, i.e., research, safety, time, and cost, the use of mini-humanoids could be essential to the research community for prototyping their research and performing tasks that are hard with fullsize humanoids.

In the direction of mini-humanoid designs, there are already some robots that have been used for humanoid research, such as 4-QRIO [19], DARwIn-OP [20], Robotis OP2/OP3 and BIOLOID, HOAP-1/2/3 from Fujitsu Lab- 


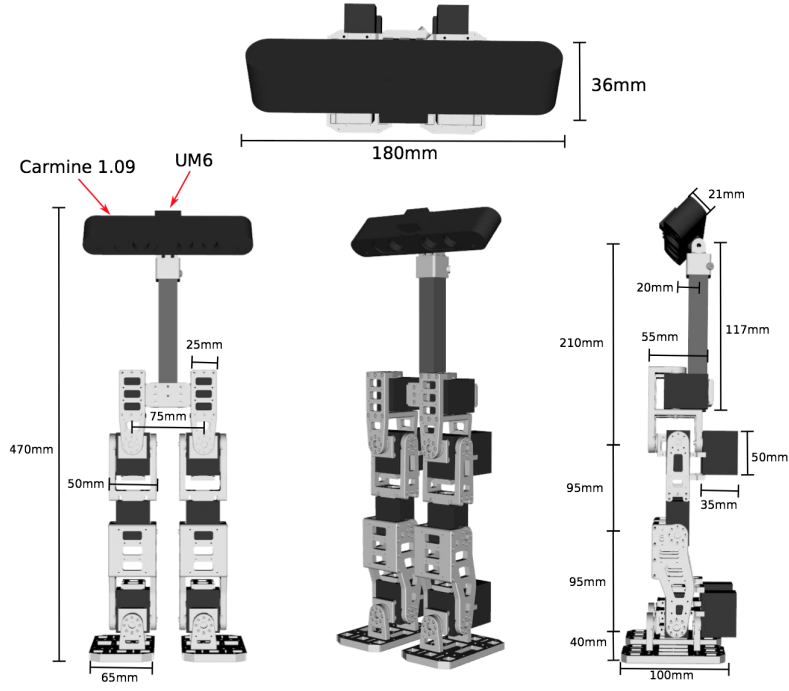

Fig. 2. The RPBP robot layout. The white parts are 3D printed.

oratories, Aldebaran NAO, and various customized ones, such as those participating in the RoboCup competition. These mini robots allowed various researchers (especially those with limited budget) to develop some very important research in the field. For instance, for locomotion purposes, which is the main interest of this paper, the NAO mini robot embedded with a range sensor on its head, was used for SLAM/navigation [21] or for state estimation during walking [22]. These methods were transferred to bigger robots, such as HRP-2. Similarly, design choices for reliable highperformance miniature humanoids were discussed in [23], while a series of mini humanoid robots (Mk.1-Mk.5 [24] and Morph3 [25]) was developed to study bipedal walking control strategies. We share the same motivation and intuition with the aforementioned works.

The cost and the design of these mini robots may vary depending on the application. With the current paper, we intend to design and build a system that is cheap (compared to other mini-humanoids such as DARwIN-OP, i.e., 12k\$), but also open-hardware and easy to repair it and replace its parts (compared to other humanoids, such as BIOLOID, for which is hard to customize it with different feet type, sensing system, etc). Moreover, we provide the open-source software packages that were developed, to further aid research in the locomotion era.

In this paper, we focus on providing an open-hardware, low-cost $(\sim 3 \mathrm{k} \$$, which comes mainly from the commercial motors) solution for the purpose of bipedal locomotion and perceptual SLAM. We designed and built the RapidPrototyped BiPed (RPBP), visualized in Fig. 1, mainly to support research in 3D perception for bipedal locomotion in uneven terrain. The term "Rapid Prototyping" refers to the 3D-printing fabrication system we used to build the majority of the robot's custom ABS plastic parts. We use an RGB-D sensor for acquiring 3D visual data, together with an Inertial Measurement Unit (IMU). The computations are taking place off-board to keep the system light and safe for big damages. Moreover, we have designed different types of feet and sensing to identify ground contact and the Center-of-Pressure (CoP) during locomotion. Last but not least, we have developed various open-source packages for environment perception that are used on the robot. We experimentally validate the usability of the robot for some simple locomotion and foot placement tasks, using SLAM from the exteroceptive perception system, and we discuss briefly the transferability of the methods to full-size humanoid robots.

We provide all the RPBP mechanical design as open-hardware on our web-site (www2.ccs.neu.edu/ research/gpc/rpbp/rpbp.html), while all the software system is implemented in $\mathrm{C}++$ (see the corresponding references in the sections below).

Next, we first provide the robot's design, including its control and perception (Sec. II). Then, we present some locomotion robotic application using the newly developed bipedal robot (Sec. III) and finally, we conclude with some future directions about our design.

\section{DESIGN}

Our intention is to develop a low-cost and open-hardware mini bipedal robot that can be used for locomotion experiments with perception. Moreover, the dimensions should be enough to complete tasks without any risk for the robot, the environment, and the human, while providing capabilities that can easily be adapted from half or full-size humanoids for real-world applications. We show the later in Sec. IIIC. In this section, we describe the platform design with its interfaces and control modules.

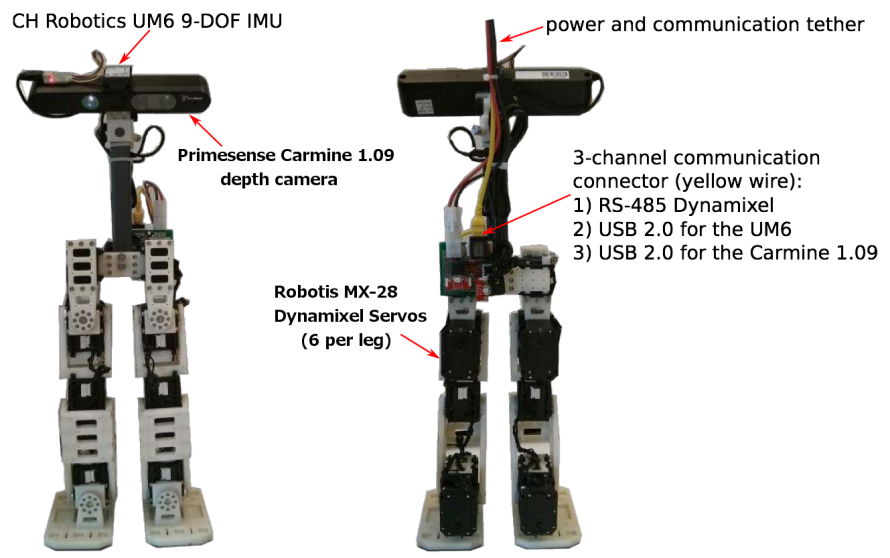

Fig. 3. The physical RPBP robot, its range/IMU sensors, actuators, and the communication system.

The robot's layout is presented in Fig. 2, while the physical hardware is shown in Fig. 3. The Rapid Prototyped BiPed (RPBP) is a twelve Degrees-of-Freedom (12-DoF) mini 3D printed robot, which stands about $47 \mathrm{~cm}$ tall and weighs about $1.7 \mathrm{~kg}$. Each of its legs has 6-DoF. The original foot type is of rectangular shape, but we show in Sec. II-B an alternative design for rock climbing and CoP sensing that we have further developed. The kinematic links and the original foot geometry have the same configuration as our full-size humanoids ones (e.g., COMAN and WALK- 


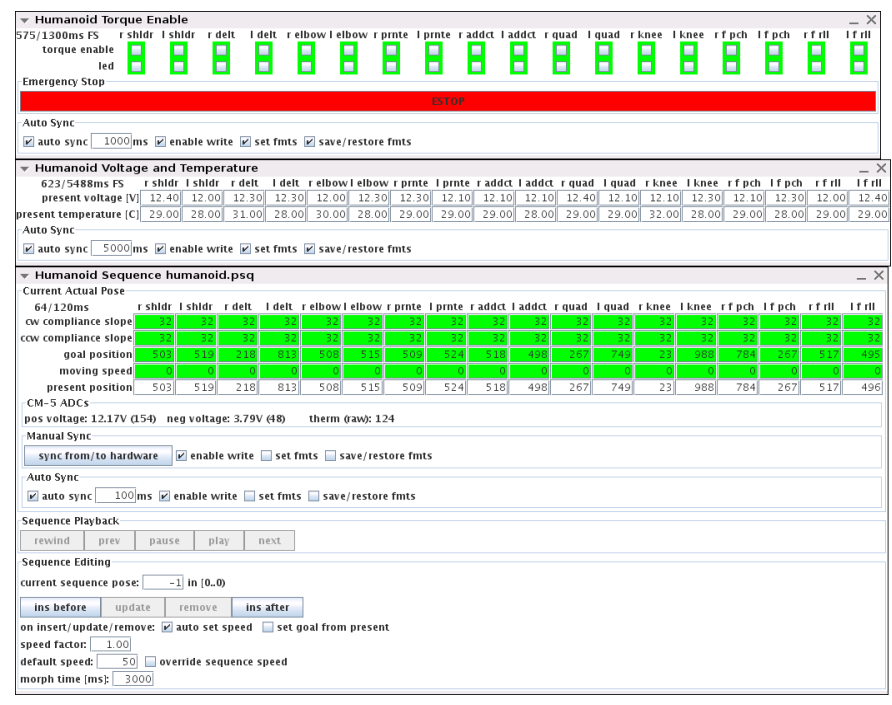

Fig. 4. The dxrobot GUI (torque enable options, voltage and temperature, servo errors, and motor sequences control).

MAN). Thus, not only we are able to perform locomotion sequences, but also the same method principles that have been developed on the mini robot can be adapted from the bigger ones. For the robot's actuation, we use the Robotis Dynamixel MX-28 servos for all joints (PID control uses the high resolution magenetic rotary encoders). Even though not a full humanoid, since RPBP has been developed also for perception research, we also include a range sensor head (Primesense Carmine 1.09) aligned with a CH Robotics UM6 IMU. Their kinematic calibration is described in Sec. IIIA. $1^{1}$. The cost of each servo is about $220 \$$, while the range and IMU sensor costs less than $430 \$$, summing up to a total cost of around $3 \mathrm{k} \$$, plus the printed plastic material.

RPBP is also a remote brain robot [26], i.e. it does not have an on-board CPU (see Fig. 3). It operates with a lightweight tether, which supplies off-board motor power and conveys sensor data to and control commands from an off-board control computer. In particular, the robot communicates with a pilot computer through a three-channel communication to control the motors (RS-485 Dynamixel) and the two USB 2.0 IMU and range sensors. The remote brain approach is not far from the full-size humanoid robots, such as our WALKMAN humanoid [?], for which we use similar wireless techniques to communicate between the robot basic on-board COM Express, with more powerful pilot PC's to perform heavy computations with the remote brain.

RPBP's mechanical design files are available on our web-page: www2.ccs.neu.edu/research/gpc/ $\mathrm{rpbp/rpbp.html.} \mathrm{It} \mathrm{includes} \mathrm{a} \mathrm{set} \mathrm{of} \mathrm{Alibre} \mathrm{CAD} \mathrm{files,}$ including STEP exports of the whole assembly and STL files (i.e., the individual 3D printable parts).

\section{A. Software Interface: dxrobot}

Since RPBP is a remote brain robot, we implemented an open-source ${ }^{2}$ Robotis Dynamixel-based communication

\footnotetext{
${ }^{1}$ The range/IMU sensors, as well as other parts of the robot, e.g. feet or actuators, are easily replaceable for the needs of the carried out research.

${ }^{2}$ BRBrain: www2.ccs.neu.edu/research/gpc/BRBrain
}

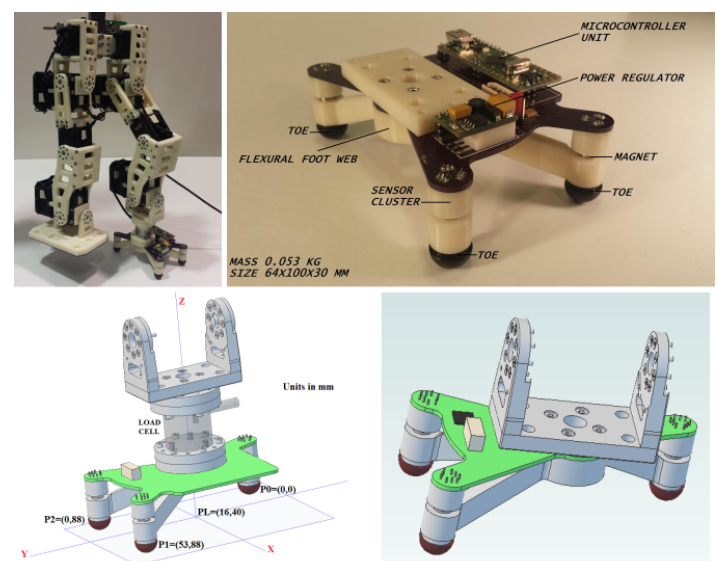

Fig. 5. The new 3-toes foot design and its microcontroller, as well as the CAD system of the foot with and without the load-cell sensor [27].

interface between the robot and the external remote computer, named dxrobot library. The software interface was originally designed in our lab to communicate with the lowcost Robotis Bioloid remote brain robot (BRBrain library), so that it can replace the limited factory-provided graphical programming system. Here we adapt/extend BRBrain for the Robotis MX-28 Dynamixel servos, through a host-based Java environment. The Java code runs on a host PC, which is used to control RPBP via a serial tether (with an option to use also a Bluetooth link). Using the dxrobot library, we implemented locomotion and stepping experiments on our mini robot.

The dxrobot is a high-level Java host control library, running on the external computer, that implements the remote brain functionality, with the data from the sensors and servos being exchanged in real-time with the host computer. The control code can be written in Java or JScheme shell for Scheme-language programming. During the constant communication with the host workstation, the 12 servos can be controlled in $100 \mathrm{~Hz}$, using the RS-485 serial cable (this allows also wireless connection, such as bluetooth, to replace the communication system). The library provides several features, such as graphical display (see Fig. 4 for a standard control GUI between the host workstation and the robot), allowing the host computer run computationally expensive code (vs. for instance DARwIN-OP robot's embedded one). The Java code that runs on the remote workstation is responsible to change the registers of the Dynamixel MX-28.

An important feature that is implemented in the dxrobot library is the ability to represent an ordered set of robot poses and pose sequences, as a set of registers on a configurable set of Dynamixel MX-28 servos. The GUI provides such capabilities and has been used to record and play-back robot stepping motions, as sequences of interpolated poses. The GUI (an interactive REPL JScheme interpreter) can be helpful in various ways, such as controlling and monitoring the servos, or generate and playback robot motion sequences (Fig. 4).

In the Sec. III we describe the high-level software packages that were used for locomotion, perception, and mapping, under the communication system that is provided by the dxrobot library. 


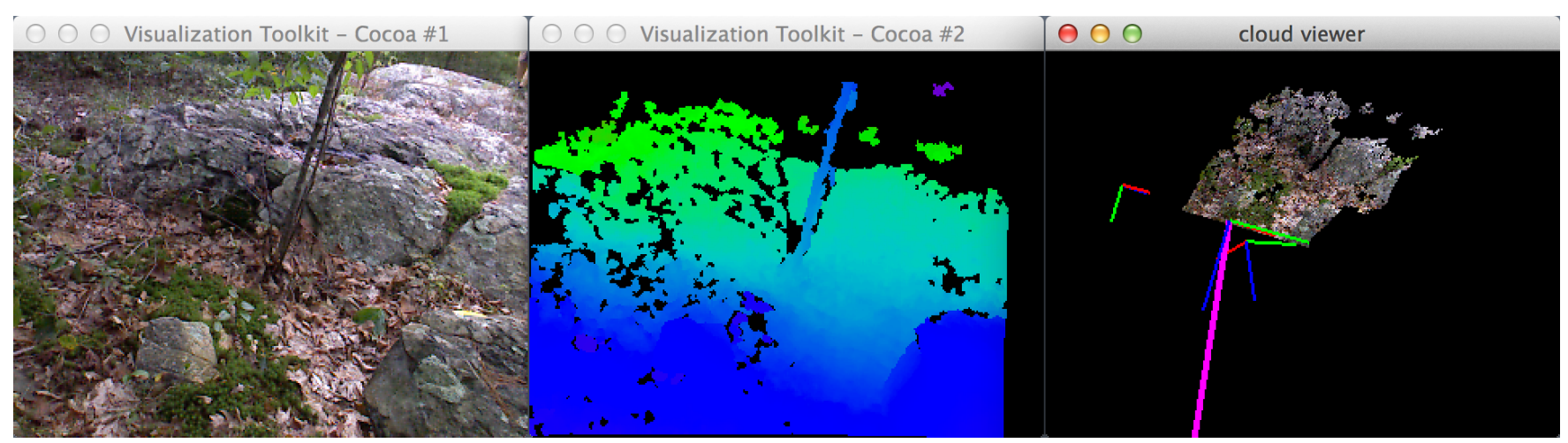

Fig. 6. IMUCam grabber with RGB, depth, and point cloud with the sensors frames (camera, IMU, global, and gravity in pink).

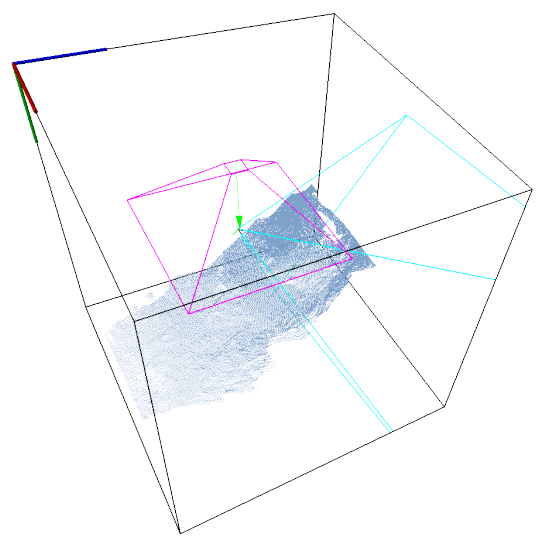

Fig. 7. The use of rxKinFu, with the 3D volume including point cloud of rough terrain, raycasted after three volume remaps, using the RPBP robot.

\section{B. Alternative Feet for Climbing}

One of the main problem that we intended to study using RBPB is stepping over rough and rocky terrain. This is a very challenging problem for bipeds, since balancing during locomotion is hard to be sustainable, and control, planning, and perception methods need to be further developed. Most of the full-size bipedal humanoid robots have flat rectangular feet. We design a new type of foot (Fig. 5) to replace RBPB robot's classic flat ones, with three toes, whose cost is around $100 \$$ and provides Hall-effect Center-of-Pressure sensing. Even though these feet are challenging during static locomotion, we believe that can be useful for research on dynamic/quasi-static stepping on rocky terrain. More details on the design and the CoP sensing, can be found in [27]. Notice that, foot forces and moments are also useful for balancing during locomotion [28], and extending our foot sensing method to measure these values is an ongoing research project.

\section{ROBOTIC APPLICATIONS}

In this section, we provide some brief information of three high-level open-source software perception packages that were developed to make RPBP robot function in the environment, the robot's control scheme, and some in-lab locomotion and stepping applications of the developed robot.

The software interface system for the high-level control of the robot is written in $\mathrm{C}++$, inheriting methods from the PCL [29] and it is open-source under our web-page.
It includes three libraries: 1) IMUCam (Sec. III-A.1), ii) rxKinFu (Sec. III-A.2), and iii) Surface Patch Library (SPL) (Sec. III-A.3).

The PCL-based IMUCam library utilizes a grabber for the range (30fps) and IMU (100fps) sensors on RPBP. The $r x K$ $i n F u$ implements a dense and real-time tracking and threedimensional mapping system [30]. The SPL implements a system for modeling and finding sets of salient bounded curved areas (i.e., patches) that fit in the environment and on the robot and are used for RPBP's stepping on rocky terrain.

\section{A. Perception System}

We first describe each software library and then we present the simple control scheme that allowed the robot to demonstrate rock stepping and locomotion motions. Last but not least, we briefly show the adaptability of the methods to our full-size humanoid robots COMAN and WALK-MAN.

1) IMUCam: The IMUCam library provides a set of registration utilities for any OpenNI-compatible range sensor (i.e., depth/RGB-D camera such as MS Kinect, ASUS, or Primesense Carmine 1.09) and CHR UM6 IMU sensors mounted on the top of the camera. The RPBP robot uses the library to grab, save, and playback 30fps RGB-D data and the corresponding time-registered 100fps IMU data. Moreover, it implements calibration between the IMU and camera sensors, using depth data of a flat surface (Fig. 6). The source-code can be found under our web-page (IMUCam: www2. ccs. neu. edu/research/gpc/imucam).

2) rxKinFu: The rxKinFu library implements a modified version of the Moving Volume KinectFusion algorithm [30]. KinectFusion has been introduced in [31] as a real-time depth-based method for dense mapping and reconstruction. Our rxKinFu library removes the limit of the original algorithm to work on relatively small volume fixed in the world, by adding volume remapping (i.e., reslicing) that allows the range sensor to move freely in the environment.

Since, the focus of RPBP is research on locomotion and rough terrain stepping, we focused on developing a package that allows it "see" the ground under its feet, through mapping and reconstruction. This is a difficult problem due to robot's legs/feet occlusion between the camera and the ground. rxKinFu library allows a volume moving freely and on-line in the environment. The volume is remapped to let 

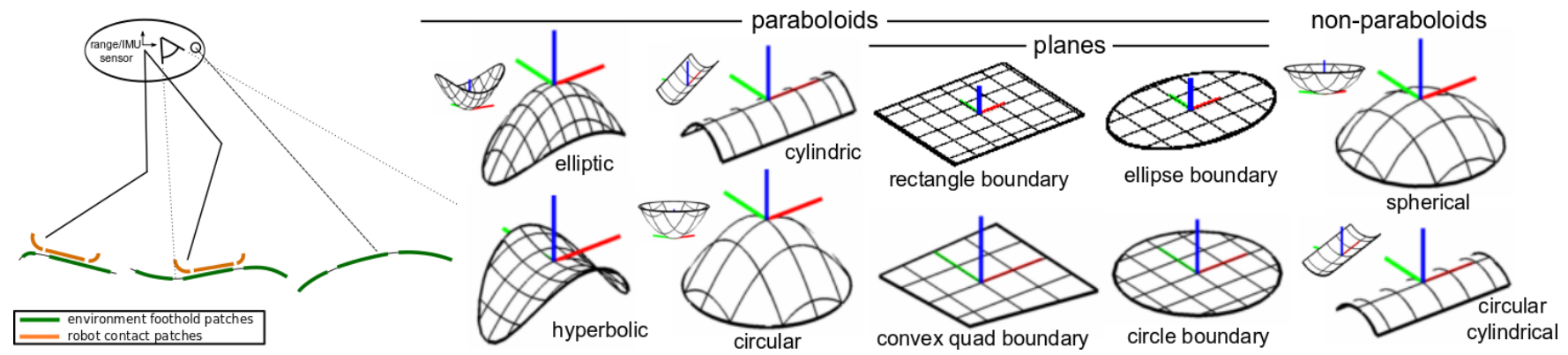

Fig. 8. The SPL, with all the curved bounded patch models (right) and the robot with patches on its foot soles and the surrounding terrain (left), used for stepping and contact reasoning.

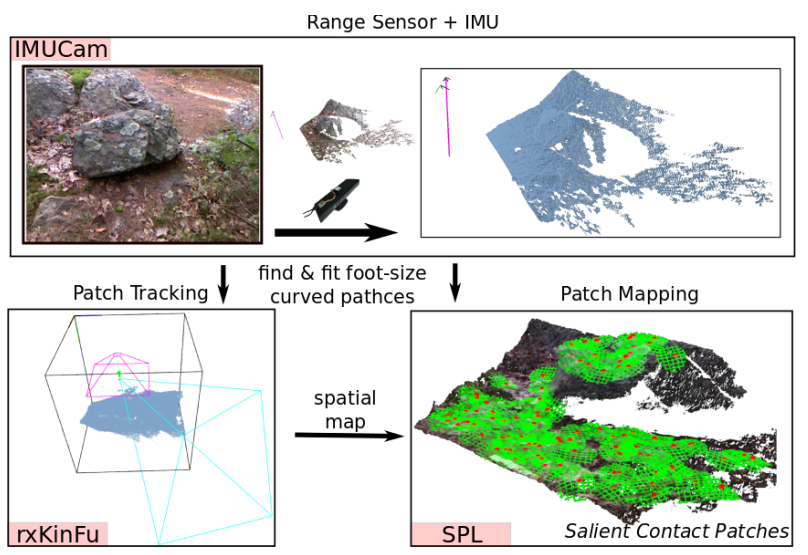

Fig. 9. Bounded patch mapping and tracking for rocky terrain, using the IMUCam, rxKinFu, and SPL software packages.

the camera pose remain fixed relative to the volume. We allow raycasted point clouds to be extracted from the volume, while the volume-to-volume transforms are maintained after each remapping. Thus, this library allows us keep track of the global camera motion and having a reconstructed spatial map of the local surrounding environment. Notice, that remapping is a heavy process that needs special care to maintain the algorithm's performance. We implemented a fast parallel method in CUDA to achieve fast performance (15fps). Fig. 7 illustrates a rough terrain after three volume remaps. Notice, that rxKinFu can be compiled with the IMUCam library to support grabbing or reading of saved depth+IMU data streams. The source-code can be found under our webpage (rxKinFu: https://github.com/RoViL-Team/ rxkinfu).

3) Surface Patch Library (SPL): The SPL [32], [33] includes a set of 10 models of foot-sized bounded curved surface patches and methods to identify salient areas in the environment, fitting methods to noisy range data, and reason about contact between patches on the robot foot sole and the surrounding areas. Uncertainty is quantified as range data covariance matrices. The patch models include seconddegree polynomials to model foot contacts with surfaces for locomotion purposes (Fig. 8). Their minimal geometric parametrization is ideal for real-time (30fps) use on the robot. The library is used together with the IMUCam and the $\mathrm{rxKinFu}$, as a complete system to apply Simultaneous Localization and Mapping (SLAM) methods that assemble on-line a map of patches for contact. In Fig. 9 the whole perception system is illustrated, in which all the developed libraries are used to map and track contact patches, potentially good for footfalls on rocky terrain [34]. In particular, depth+IMU data streams are provided from the IMUCam to the rxKinFu reconstruction and tracking system, whose output is the input of the soft real-time SPL method to find and fit foot-size patches. The source-code can be found under our web-page (SPL: http://dkanou.github. io/projects/spl).

\section{B. Control System for RPBP Locomotion and Rock Stepping}

For testing the ability of the RPBP robot to perform some basic locomotion and stepping tasks, we developed two forms of motion control. First, we implemented an openloop manually tuned gait for flat terrain navigation (Fig. 10left). We let the robot locomote in loops on a table, while we were running the rxKinFu patch tracking and the SPL patch mapping algorithms, to verify the ability of the robot to use the libraries with the integrated 3D perception into the bipedal locomotion.

Then, in [34] we implemented a basic patch-based control method for rock stepping. Having fitted a set of visible curved patches in the space around the robot (as in Fig. 9), we compare them with a pre-defined database of known patches paired with corresponding pre-defined step motions on rocks (trained with the use of our dxrobot software interface GUI, introduced in Sec. II-A). RPBP robot is then able to identify and step on such rocks. Notice, that the robot is statically stable once the stepping foot has settled on the rock and closed-loop balancing is under development. As shown in Fig. 10-right, the rock stepping control scheme includes: 1) the RPBP robot model with the dxrobot interface and 2) the RPBP walk control library of motion sequences related to patches. Videos of the implemented control systems can be found under our web-page: www2 . CCS . neu . edu/research/gpc/rpbp/rpbp.html.

\section{Transfer of Knowledge to Half/Full-Size Humanoids}

Given that we managed to make the developed RPBP mini robot to step on rocks and use the software interface to achieve this type of locomotion control as a prototype method, we have tested the ability to use the same system with a different controller for locomotion research on half and full-size humanoids, such as COMAN [8] and WALKMAN [16]. Transfer of knowledge from small to big robotic systems is a challenging task and the methodology needs 


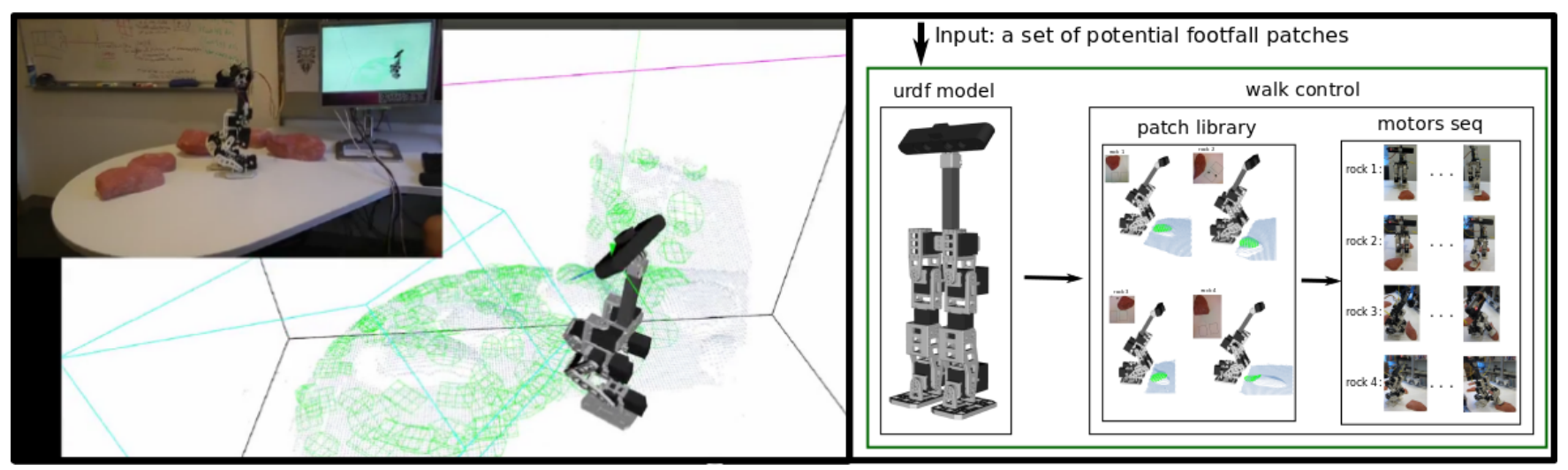

Fig. 10. Left: open-loop locomotion of RPBP, which is manually tuned gait for flat terrain, while detecting and mapping curved surface patches using real-time 3D perception. Right: control method to step on rocks, using 3D perception to select pre-defined motion plans [34].

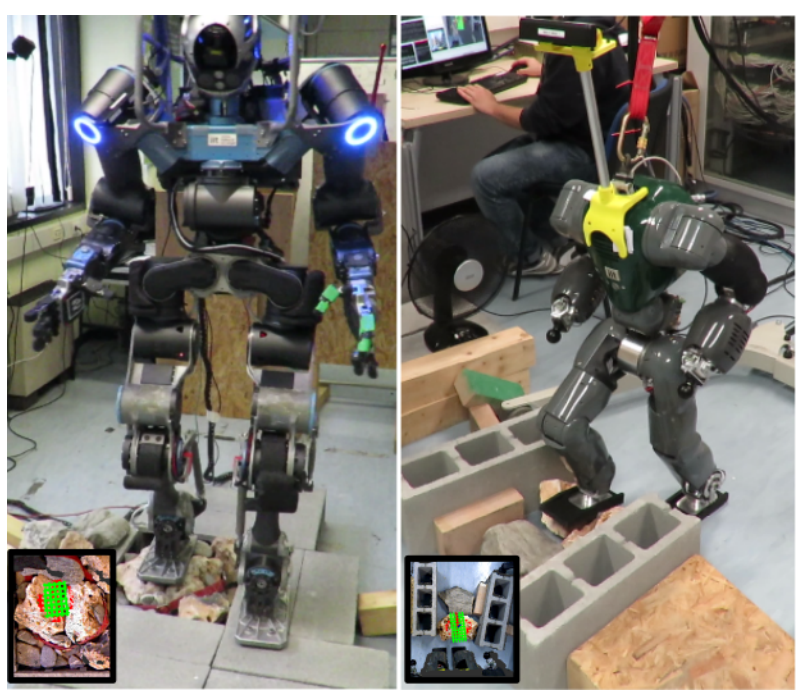

Fig. 11. WALK-MAN and COMAN humanoid robots, using SPL for finding contact patches (in green) onto rocks and stepping on them [18].

careful consideration. Here, we do not intend to fully describe this methodology, which by itself is an active research topic, but to present a proof-of-concept. As mentioned before when trying locomotion methods directly on big robots there are several risks and difficulties, such as damages or robot management (with the crane, etc.). On the other side, when using a small robot to achieve such a task, the usability improves since one can run several experiments without the risks and technical difficulties. The key issue while transferring the knowledge then is the fact that there are major differences in the hardware (motors, sensors, weight, torques, etc.) depending on their size, i.e. the bigger the robot is the more difficult it is to control it.

To solve this issue, we split our total architecture to black boxes, where we treat the developed perception-only system as a stand-alone unit. In this way we let the control, stabilization, and trajectory planning systems to vary depending on the robot. In particular, to transfer the perception module of rock stepping from the RPBP to our COMAN and WALKMAN robots, we firstly used the patch fitting method and the patch mapping system, as described above, to detect partial foot contacts with the environment [18]. The only changing parameter between the mini and the full-size robot is the foot sole size. Then, we used our specialized for big bipeds control system, with its inverse kinematics/dynamics and stabilization methods [16], to make COMAN and WALKMAN step on rocky terrain with flat feet (Fig. 11).

\section{CONCLUSIONS}

This paper presented the design of a novel mini-bipedal robot, called RPBP. The robot is designed to help with research on bipedal locomotion, using 3D perception. We provided a brief description of the software firmware dxrobot, for the communication between the remote brained robot and the host workstation. Moreover, we presented three opensource packages (IMUCam, rxKinFu, and SPL) that accompany the robot and are used for perception, mapping, and tracking. We finally demonstrated that the whole integrated system is able to achieve some basic control motions for walking on flat terrain and performing some rock stepping using vision. We used a tweaked version of the methods on full-size humanoids for stepping over rocks to show the knowledge transferability between the mini-biped and the half or full-size humanoids.

We plan in continuing the implementation of more complex control motion on the robot (inverse kinematic/dynamics and stabilizers), using also the new 3-toes foot design. Moreover, we plan in using the mini-biped RPBP for different types of research, for instance on humanoid falling. Last but not least, we intend to design and attach an upper-body to the robot for a more human-like design as other conventional mini robotic platforms. All the hardware and software packages that are described in this paper are released as open, under our web-page.

\section{ACKNOWLEDGEMENT}

This work is supported by the CogIMon (grant no. 644727) EU project and the National Science Foundation (grant no. 1149235). The Titan Xp was donated by the NVIDIA Corporation.

\section{REFERENCES}

[1] S. Sugano and I. Kato, "WABOT-2: Autonomous Robot with Dexterous Finger-Arm-Finger-Arm Coordination Control in Keyboard Performance," in IEEE ICRA, 1987, pp. 90-97.

[2] M. Hirose and Y. Haikawa, "Development of Humanoid Robot ASIMO," in IEEE/RSJ IROS, 2001, pp. 1-6. 
[3] K. Kaneko, F. Kanehiro, M. Morisawa, K. Akachi, G. Miyamori, A. Hayashi, and N. Kanehira, "Humanoid Robot HRP-4 - Humanoid Robotics Platform with Lightweight and Slim Body," in IEEE/RSJ IROS, 2011, pp. 4400-4407.

[4] I.-W. Park, J.-Y. Kim, and J.-H. Oh, "Mechanical Design of the Humanoid Robot Platform HUBO," Journal of Advanced Robotics, vol. 21, no. 11, pp. 1305-1322, 2007.

[5] Y. Ito, T. Nakaoka, J. Urata, Y. Nakanishi, K. Okada, and M. Inaba, "Design and Development of a Tendon-Driven and Axial-Driven Hybrid Humanoid Leg with High-Power Motor Driving System," in IEEE-RAS ICHR, 2012, pp. 475-480.

[6] S. Lohmeier, T. Buschmann, H. Ulbrich, and F. Pfeiffer, "Modular Joint Design for Performance Enhanced Humanoid Robot LOLA," in IEEE ICRA, 2006, pp. 83-93.

[7] A. Parmiggiani et al., "The Design of the iCub Humanoids Robot," IJHR, vol. 09, no. 04, 2012.

[8] N. G. Tsagarakis et al., "Compliant Humanoid COMAN: Optimal Joint Stiffness Tuning for Modal Frequency Control," in IEEE ICRA, 2013, pp. 673-678.

[9] N. Paine et al., "Actuator Control for the NASA-JSC Valkyrie Humanoid Robot: A Decoupled Dynamics Approach for Torque Control of Series Elastic Robots," JFR, vol. 32, no. 3, pp. 378-396.

[10] J. Englsberger et al., "Overview of the torque-controlled humanoid robot toro," in 14th ICHR, 2014, pp. 916-923.

[11] J. Pratt et al., "Capturability-based Analysis and Control of Legged Locomotion, Part 1: Theory and Application to Three Simple Gait Models," IJRR, vol. 31, no. 9, pp. 1094-1113, 2012.

[12] K. Kojima et al., "Development of Life-Sized High-Power Humanoid Robot JAXON for Real-World Use," in IEEE-RAS ICHR, Nov 2015, pp. 838-843.

[13] H. Kaminaga et al., "Mechanism and Control of Whole-Body ElectroHydrostatic Actuator Driven Humanoid Robot Hydra," in ISER, 2016, pp. 656-665.

[14] K. Coleman et al., "Team VALOR's ESCHER: A Novel Electromechanical Biped for the DARPA Robotics Challenge," JFR, vol. 34, no. 5, pp. 912-939, 2017.

[15] O. Stasse et al., "TALOS: A new humanoid research platform targeted for industrial applications," in IEEE-RAS ICHR, 2017.

[16] N. G. Tsagarakis et al., "WALK-MAN: A High-Performance $\mathrm{Hu}-$ manoid Platform for Realistic Environments," JFR, vol. 34, no. 7, pp. 1225-1259, 2017.

[17] J. Lee, W. Choi, D. Kanoulas, R. Subburaman, D. G. Caldwell, and
N. G. Tsagarakis, "An Active Compliant Impact Protection System for Humanoids: Application to WALK-MAN Hands," in IEEE-RAS ICHR, 2016, pp. 778-785.

[18] D. Kanoulas, C. Zhou, A. Nguyen, G. Kanoulas, D. G. Caldwell, and N. G. Tsagarakis, "Vision-based foothold contact reasoning using curved surface patches," in IEEE-RAS ICHR, 2017, pp. 121-128.

[19] T. Ishida, "A Small Biped Entertainment Robot SDR-4X II," in IEEE IROS, vol. 3, 2003, pp. 1046-1051.

[20] I. Ha, Y. Tamura, H. Asama, J. Han, and D. W. Hong, "Development of Open Humanoid Platform DARwIn-OP," in SICE, 2011, pp. 21782181.

[21] D. Maier, C. Lutz, and M. Bennewitz, "Integrated Perception, Mapping, and Footstep Planning for Humanoid Navigation among 3D Obstacles," in IEEE/RSJ IROS, 2013, pp. 2658-2664.

[22] S. Piperakis, M. Koskinopoulou, and P. Trahanias, "Non-Linear State Estimation for Humanoid Robot Walking," in IEEE RA-L, 2018.

[23] T. Sugihara, K. Yamamoto, and Y. Nakamura, "Hardware Design of High Performance Miniature Anthropomorphic Robots," RAS, vol. 56, no. 1, pp. 82-94, 2008.

[24] T. Furuta, T. Tawara, Y. Okumura, M. Shimizu, and K. Tomiyama, "Design and Construction of a Series of Compact Humanoid Robots and Development of Biped Walk Control Strategies," RAS, vol. 37, no. 2, pp. 81-100, 2001.

[25] T. Tawara and other, "Morph: A Desktop-Class Humanoid Capable of Acrobatic Behavior," IJRR, vol. 23, no. 10-11, pp. 1097-1103, 2004.

[26] M. Inaba, "Remote-brained Robots," in IJCAI, 1997, pp. 1593-1606.

[27] S. C. Gomez, M. Vona, and D. Kanoulas, "A Three-Toe Biped Foot with Hall-Effect Sensing," in IEEE/RSJ IROS, 2015, pp. 360-365.

[28] S. Kajita et al., "Biped Walking Stabilization Based on Linear Inverted Pendulum Tracking," in IEEE/RSJ IROS, 2010, pp. 4489-4496.

[29] R. B. Rusu and S. Cousins, "3D is here: Point Cloud Library (PCL)," in ICRA, 2011.

[30] H. Roth and M. Vona, "Moving Volume KinectFusion," in BMVC, 2012.

[31] R. A. Newcombe et al., "KinectFusion: Real-Time Dense Surface Mapping and Tracking," in IEEE ISMMAR, 2011.

[32] D. Kanoulas and M. Vona, "The Surface Patch Library (SPL)," in IEEE ICRA Workshop, 2014, dkanou.github.io/projects/spl/.

[33] M. Vona and D. Kanoulas, "Curved Surface Contact Patches with Quantified Uncertainty," in IEEE/RSJ IROS, 2011, pp. 1439-1446.

[34] D. Kanoulas, "Curved Surface Patches for Rough Terrain Perception," Ph.D. dissertation, CCIS, Northeastern University, August 2014. 